Departamento de Anatomía Patológica. Facultad de Medicina. Universidad de La Frontera. Temuco, Chile.

BIOREN (Scientific and Technological Bioresource Nucleus).

Recibido el 30 de septiembre de 2013, aceptado el 7 de abril de 2014.

Correspondencia a: Dr. Oscar Tapia E. Departamento de Anatomía Patológica, Facultad de Medicina, Universidad de La Frontera. Manuel Montt 112 Teléfono: 045-296530. Código Postal 478-1176. Temuco, Chile. otescalona@gmail.com

\section{Apendicitis granulomatosa sarcoidal: una infrecuente entidad clínico-patológica. Caso clínico}

\author{
OSCAR TAPIA E.
}

\section{Granulomatous appendicitis caused by sarcoidosis. Report of one case}

Granulomatous appendicitis is uncommon and can be caused by Crohn's disease, foreign body reactions, infections or sarcoidosis. We report a 26-year-old female consulting in the emergency room for pain in the right lower abdomen. She was released with analgesics and consulted 14 days later for the same pain. This time she was operated with the preoperative diagnosis of appendicitis. The pathological study of the surgical piece was compatible with a granulomatous appendicitis caused by sarcoidosis.

(Rev Med Chile 2014; 142: 526-528)

Key words: Appendicitis; Sarcoidosis.
I a apendicitis granulomatosa es una entidad clínica poco frecuente $(0,1-2 \%)$ considerándose dentro de los diagnósticos histopatológicos diferenciales a la enfermedad de Crohn, reacciones por cuerpo extraño, infecciones (bacterianas, micóticas y parasitarias) y la sarcoidosis ${ }^{1-9}$.

La sarcoidosis por su parte corresponde a una enfermedad granulomatosa multisistémica que clásicamente afecta a los pulmones, ganglios linfáticos y piel; menos frecuentemente existe compromiso ocular, hepático, esplénico, óseo, muscular, cardiaco, del sistema nervioso y gastrointestinal. A nivel gastrointestinal en tanto, el compromiso es habitualmente asintomático, reportándose afectación del esófago, estómago e intestino, mientras que el compromiso apendicular es extremadamente infrecuente con aislados reportes de $\operatorname{casos}^{2,9,10-18}$.

Dado el infrecuente compromiso apendicular, presentamos el caso de una mujer de 26 años, sometida a apendicectomía por sospecha clínica de apendicitis aguda y en la que el estudio histopatológico de la pieza quirúrgica es compatible con una apendicitis granulomatosa sarcoidal.

\section{Caso clínico}

Mujer de 26 años sin antecedentes de importancia que consultó en servicio de urgencia refiriendo dolor en hemi-abdomen inferior desde hacía aproximadamente $24 \mathrm{~h}$; no asociado a vómitos ni diarrea. Al examen físico abdominal se constató un abdomen sensible a la palpación de la fosa iliaca derecha, sin signos de irritación peritoneal. La temperatura axilar y rectal fue $36,9^{\circ} \mathrm{C}$ y $37,4^{\circ} \mathrm{C}$ respectivamente, sin alteraciones en el hemograma (leucocitos 8.200) y proteína $\mathrm{C}$ reactiva de 5 . La evaluación ginecológica resultó normal y test de embarazo negativo. Dado los hallazgos clínicos y de laboratorio se indicó tratamiento sintomático con analgésicos y antiespasmódicos.

La paciente consultó luego de 14 días por presentar nuevamente dolor abdominal asociado a sensación febril. El examen físico constató temperatura axilar de $37,7^{\circ} \mathrm{C}$ y rectal de $38,2^{\circ} \mathrm{C}$ con dolor a la palpación de la fosa ilíaca derecha. El hemograma mostró recuento de leucocitos de 10.600 , velocidad de eritrosedimentación de 9 y proteína C reactiva 18 . Con la sospecha de 

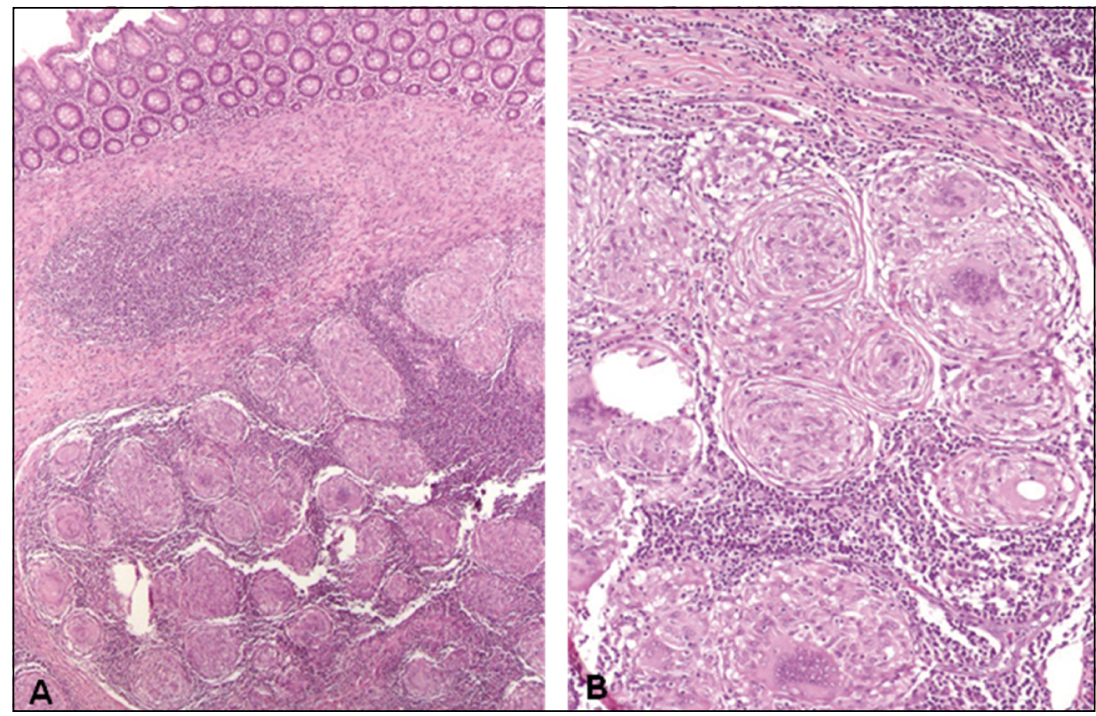

Figura 1. (HE, A 40X y B 100X). Pared apendicular con granulomas no caseosos de bordes bien delimitados con ausencia de corona linfocitaria. No se observó elementos bacterianos, micóticos ni parasitarios. apendicitis aguda se realizó apendicetomía, sin complicaciones quirúrgicas.

El estudio macroscópico de la pieza quirúrgica demostró un apéndice cecal de $6,7 \mathrm{~cm}$ de longitud y $1,1 \mathrm{~cm}$ de diámetro. La superficie serosa y mucosa no presentó lesiones. El examen microscópico reveló la presencia de granulomas epiteloídeos no caseificantes de bordes bien definidos, con algunas células gigantes tipo Langhans y ausencia de corona linfocitaria. Se constataron escasos cuerpos asteroides, sin evidenciarse cuerpos de Schaumann (conchoides) ni de Hamazaki-Wesenberg. No se identificó inflamación aguda asociada ni material extraño en el espesor de la pared apendicular (Figura 1). Las tinciones histoquímicas para microorganismos (Ziehl Neelsen, Gram, PAS, Grocott y Warthin starry) y PCR para Mycobacterium tuberculosis resultaron negativas. Los hallazgos histopatológicos fueron compatibles con una apendicitis granulomatosa sarcoidal.

La paciente evolucionó sin complicaciones post-operatorias. En control al $8^{\circ}$ mes la radiografía de de tórax, colonoscopía y tomografía computada (TC) de tórax-abdomen y pelvis resultaron normales, encontrándose luego de 13 meses asintomática.

\section{Discusión}

La apendicitis granulomatosa constituye una infrecuente entidad histopatológica, identificada en tan sólo $0,1-2 \%$ de las piezas de apendicectomía; considerando su diagnóstico diferencial a la enfermedad de Crohn, reacciones por cuerpo extraño e infecciones bacterianas (Mycobacterium tuberculosis, Yersiniosis, Actinomycosis, Brucella y Campylobacter), parasitarias (Enterobius vermicularis, schistosoma) y fúngicas (Histoplasmosis, Blastomycosis, Candidiasis) ${ }^{1-9}$.

La sarcoidosis en tanto corresponde a una enfermedad granulomatosa sistémica de etiología desconocida caracterizada histológicamente por la formación de granulomas no caseosos que afectan diferentes órganos ${ }^{9,10,12}$. El compromiso pulmonar se observa en más de $90 \%$ de los pacientes con sarcoidosis mientras que la afectación gastrointestinal se ha reportado en tan sólo 10\% de estos sujetos; pudiendo afectar a este nivel el esófago, estómago, intestino delgado y grueso. Habitualmente este compromiso es asintomático y tan sólo en $0,6 \%$ de los pacientes con compromiso GI se hace clínicamente manifiesto. La sarcoidosis apendicular en tanto es extremadamente rara, identificándose en una revisión que incluyó 50.000 apendicectomías tan sólo un caso de apendicitis granulomatosa ${ }^{3,9,12,13,19,20}$.

Si bien habitualmente el compromiso gastrointestinal es asintomático, el caso reportado se presentó con dolor abdominal recurrente y simulando una apendicitis aguda; sin demostrar el estudio anatomo-patológico inflamación aguda asociada, representando esta una forma excepcio- 
nal de presentación de la sarcoidosis con escasos reportes en la literatura. La patogenia del dolor abdominal en estos casos no es muy clara y se especula que la inflamación granulomatosa produciría fibrosis y obstrucción del lumen apendicular con el consiguiente dolor, de tipo recurrente, progresivo y en ocasiones cólico ${ }^{12,16,21}$.

En esta paciente el estudio histopatológico con técnicas complementarias para descartar una probable etiología infecciosa resultaron negativas y en los posteriores controles el estudio con colonoscopía, radiografía de tórax y TC de tórax-abdomen y pelvis permitieron descartar otras enfermedades granulomatosas o condiciones asociadas.

El tratamiento en los sujetos con sarcoidosis considera la terapia con corticosteroides, dependiendo la duración de este tratamiento de la respuesta clínica, observándose recurrencia de la enfermedad en casos de rápida reducción de la terapia corticoesteroidal. El uso de metotrexato, clorambucil, azatioprina, infliximab y ciclosporina son fármacos alternativos en casos refractarios a la prednisona ${ }^{9}$.

Finalmente, podemos concluir que la apendicitis granulomatosa sarcoidal representa una infrecuente entidad histopatológica que debe ser diferenciada de otras enfermedades granulomatosas infecciosas e inflamatorias, dado el distinto curso clínico, tratamiento y complicaciones asociadas a cada una de ellas; debiendo, por tanto, el anatomo-patólogo estar entrenado para realizar un adecuado diagnóstico que permita al médico clínico instaurar el tratamiento adecuado.

\section{Referencias}

1. Blair NP, Bugis SP, Turner LG, MacLeod MM. Review of the pathological diagnosis of 2215 appendicectomy specimens. Am J Surg 1993; 165: 618-20.

2. Tucker O, Healy V, Jeffers M, Keane F. Granulomatous appendicitis. Surg J R Coll Surg Edinb Irel 2003; 1: 2869.

3. Collins DCS. A study of 50,000 specimens of the human vermiform appendix. Surg Gynecol Obstet 1995; 101: 437-45.

4. Ojo OS, Udeh SC, Odesanmi WO. Review ofthe histopathological findings in appendices removed for acute apendicitis in Nigerians. J R Coll Surg Edinb 1991; 36: 245-8.
5. Van Noyen R, Selderslaghs R, Bekaert J, Wauters G, Vandepitte J. Causative role of Yersinia and other enteric pathogens in the apendicular síndrome. Eur J Clin Microbiol Infect Dis 1991; 10: 735-41.

6. Moyana TN, Kulaga A, Xiang J. Granulomatous apendicitis in acute myeloblastic leukemia: expanding the clinicopathologic spectrum of invasive candidiasis. Arch Pathol Lab Med 1996; 120: 203-5.

7. Adebamowo CA, Akang EE, Ladipo JK, Ajao OG. Schistosomiasis of the appendix. Br J Surg 1991; 78: 1219-21.

8. Baleh CM, Silver D. Foreign bodies in the appendix: report of eight cases and review of the literatura. Arch Surg 1971; 102: 14-20.

9. Vahid B, Spodik M, Braun K, Ghazi L, Esmaili A. Sarcoidosis of Gastrointestinal Tract: A Rare Disease. Dig Dis Sci 2007; 52: 3316-20.

10. Beniwal R, Cummings O, Cho W. Symptomatic gastrointestinal sarcoidosis: Case report and review of the literature. Digestive Diseases and Sciences 2003; 48: 174-8.

11. Stampfl DA, Grimm IS, Barbout DJ, Rosata FE, Gordon CJ. Sarcoidosis causing duodenal obstruction. Case report and review of gastrointestinal manifestations. Dig Dis Sci 1990; 35: 526-32.

12. Cullinane D, Schultz S, Zellos L, Holt R. Sarcoidosis Manifesting as Acute Appendicitis. Report of a Case. Dis Colon Rectum 1997; 40: 109-11.

13. Healy V, Jeffers M, Keane FBV, Tucker ON. Granulomatous appendicitis. J Roy Coll Surg Edinb 2003; 1: 286-9.

14. Clarke H, Pollett W, Chittal S. Sarcoidosis with involvement of the appendix. Arch Intern Med 1983; 142: 1603-4.

15. Shienfeld WT, Rubinow M. Noncaseating epitheloid granuloma of the appendix (localized sarcoid disease?). J Int Coil Surg 1964; 42: 1-4.

16. Macleod IB, Jenkins AM, Gill W. Sarcoidosis involving the vermiform appendix. J R Coll Surg Edinb 1965; 10: 319-23.

17. Munt PW. Sarcoidosis of appendix presenting as appendiceal perforation and abscess. Chest 1974; 66: 295-7.

18. Bystrom J. Localized sarcoidosis of the appendix simulating Mb Crohn. Acta Chir Scand 1968; 134: 163-5.

19. Fanburg BL. Sarcoidosis and other granulomatous diseases of the lung. New York: Marcel Dekker, 1983.

20. Israel HL, Sones M. Sarcoidosis: clinical observation on one hundred sixty cases. Arch Intern Med 1953; 102: 766-76.

21. McPherson JG, Yeoh CB. Rare manifestations of sarcoidosis. J Natl Med Assoc 1993; 85: 869-72. 